\title{
Erratum to: Risks of permanent disability in low back pain patients associated with different job positions: a 5-year follow-up study
}

\author{
Thomas Maribo $^{1,2,5} \cdot$ Berit Schiøttz-Christensen ${ }^{3} \cdot$ Chris Jensen $^{4}$ Lone Donbæk Jensen ${ }^{2}$
}

Published online: 19 October 2015

(c) Springer-Verlag Berlin Heidelberg 2015

\section{Erratum to: Eur Spine J DOI 10.1007/s00586-015-4118-4}

Due to an error a wrong Abstract was published

The correct Abstract should be:

\begin{abstract}
Purpose This study aimed at providing prognostic information on vocational outcome in a large cohort of LBP patients with a specific focus on predictors of permanent disability benefits.

Methods We included 5945 LBP patients referred to Aarhus University Hospital during 2001-2005 and followed them for 5 years. On the basis of membership of an unemployment insurance fund patients were divided into five groups defining job position. Weekly information on unemployment benefit, sickness benefit, permanent
\end{abstract}

The online version of the original article can be found under doi:10.1007/s00586-015-4118-4.

Thomas Maribo

thomas.maribo@stab.rm.dk

1 Rehabilitation Center Marselisborg, Department of Public Health, Section of Social Medicine and Rehabilitation, Aarhus University, Bygning 1B, P. P. Ørumsgade 11, 8000 Aarhus C, Denmark

2 Public Health and Quality Improvement, Central Denmark Region, Aarhus, Denmark

3 Spine Centre of Southern Denmark, Hospital Lillebaelt, Middelfart, Denmark

4 National Centre for Occupational Rehabilitation, Rauland, Norway

5 Department of Occupational Medicine, Danish Ramazzini Center, Aarhus University Hospital, Aarhus, Denmark disability benefits, and other social benefits were obtained during the 5 years of follow-up from a national register. Significant predictors of vocational outcome 5 years after LBP diagnosis were identified by multivariate Cox proportional hazard modeling.

Results A pronounced difference in the risk of permanent disability benefits between various job positions were found, with an adjusted hazard ratio of 5.66 for blue-collar workers compared to managers and the highly educated. One year after hospital contact $5.9 \%$ of the population was on permanent disability benefit; this number increases to $25.1 \%$ after 5 years.

Conclusions Different job positions marked a difference in risk of permanent disability benefit; with blue-collar workers at high risk compared to managers and the highly educated. 